\title{
P02-15
}

\section{EFFICACY OF COMBINED TREATMENTS, PSYCHOLOGICAL AND PHARMACOLOGICAL, FOR DEPRESSION IN ADULTS}

M. Carvalho ${ }^{1,2}$, D. Estevens ${ }^{1}$, O. Tur ${ }^{1}$

${ }^{1}$ Psychiatry Unit, CHBA, Portimão, ${ }^{2}$ Faculty of Psychology, ULHT, Lisbon, Portugal

According to the state of the art, combined treatments for depression are more efficacious than a unique approach. However studies on the efficacy of different psychological treatments are scarce. We will present the results of an on-going research, carried out with the main purpose of analysing the efficacy of two psychological empirically validated treatments for depression, CognitiveBehavioral Therapy (CBT) and Interpersonnal Therapy (IPT), combined with psychopharmacological approach. A sample of 30 adult females will be randomly assigned to one of three conditions: 1) CBT and pharmacological treatment; 2) IPT and pharmacological treatment; and 3) only pharmacological treatment. A multimodal assessment matrix will be used to evaluate depression, suicidal ideation and intention, and individual, social, and environmental factors commonly related to the onset of major depression, namely hopelessness and pessimism, cognitive processing, interpersonal relations, life events, before, at the end of treatments, and at follow-up. We expect that both combined approaches will be more efficacious in the reduction of depressive symptoms, including suicidal behaviours, compared to the pharmacological approach. We also expect to understand the sensitivity to change of the assessed psychological processes underlying each form of psychotherapy. 